\title{
Advances in Multiferroic Nanomaterials Assembled with Clusters
}

\begin{abstract}
Shifeng Zhao
School of Physical Science and Technology and Inner Mongolia Key Lab of Nanoscience and Nanotechnology, Inner Mongolia University,
\end{abstract} Hohhot 010021, China

Correspondence should be addressed to Shifeng Zhao; zhsf@imu.edu.cn

Received 17 June 2014; Accepted 28 October 2014

Academic Editor: Daniela Predoi

Copyright (C) 2015 Shifeng Zhao. This is an open access article distributed under the Creative Commons Attribution License, which permits unrestricted use, distribution, and reproduction in any medium, provided the original work is properly cited.

\begin{abstract}
As an entirely new perspective of multifunctional materials, multiferroics have attracted a great deal of attention. With the rapidly developing micro- and nano-electro-mechanical system (MEMS\&NEMS), the new kinds of micro- and nanodevices and functionalities aroused extensive research activity in the area of multiferroics. As an ideal building block to assemble the nanostructure, cluster exhibits particular physical properties related to the cluster size at nanoscale, which is efficient in controlling the multiferroic properties for nanomaterials. This review focuses on our recent advances in multiferroic nanomaterials assembled with clusters. In particular, the single phase multiferroic films and compound heterostructured multiferroic films assembled with clusters were introduced detailedly. This technique presents a new and efficient method to produce the nanostructured multiferroic materials for their potential application in NEMS devices.
\end{abstract}

\section{Introduction}

Multiferroics have attracted increasing attention due to simultaneous coexistence of ferromagnetic, ferroelectric, or ferroelastic ordering $[1,2]$. Many researchers focused on the magnetoelectric effect driven by the prospect of controlling polarization by magnetic field and magnetization by electrical field [3], which opens up an entirely new perspective of magnetic/ferroelectric data storage media, spin-based devices (spintronics), magnetocapacitive devices, magnetic sensors, nonvolatile memories, random access memory, and so forth [4-8]. Since its discovery a century ago, ferroelectricity has been linked to the ancient phenomena of magnetism. Attempts to combine the dipole and spin orders into one system started in the 1960s in $\mathrm{Cr}_{2} \mathrm{O}_{3}$ single crystal $[9,10]$, and other single phase multiferroics, including boracites $\left(\mathrm{Ni}_{3} \mathrm{~B}_{7} \mathrm{O}_{13} \mathrm{I}, \mathrm{Cr}_{3} \mathrm{~B}_{7} \mathrm{O}_{13} \mathrm{Cl}\right)$ [10], fluorides $\left(\mathrm{BaMF}_{4}, \mathrm{MMn}, \mathrm{Fe}\right.$, $\mathrm{Co}, \mathrm{Ni})$ [11, 12], magnetite $\mathrm{Fe}_{3} \mathrm{O}_{4}$ [13], (Y/Yb) $\mathrm{MnO}_{3}$ [14], and $\mathrm{BiFeO}_{3}$ [15], were identified in the following decades. However, such a combination in these multiferroics has been proven to be unexpectedly tough.
Moreover, by growing composite films combined with piezoelectric and magnetostrictive materials, the strong magnetoelectric coupling effect could be achieved due to product property. Much work has been done to prepare the composite films by combining perovskite ferroelectric oxides (e.g., $\mathrm{Pb}\left(\mathrm{Zr}_{0.52} \mathrm{Ti}_{0.48}\right) \mathrm{O}_{3}(\mathrm{PZT}), \mathrm{BaTiO}_{3}$ ) with ferromagnetic oxides (e.g., $\mathrm{CoFe}_{2} \mathrm{O}_{4}, \mathrm{La}_{0.67} \mathrm{Sr}_{0.33} \mathrm{MnO}_{3}$ ) [16-21]; however, due to the low magnetostriction of these ferromagnetic oxides and Ni metal, the reported magnetoelectric effects in these composite films are generally not strong.

As well known, rare earth iron alloy $(\mathrm{R}-\mathrm{Fe}, \mathrm{R}=$ rare earth element) possesses giant magnetostriction, being an order of magnitude greater than the ferromagnetic oxides [22]. The previous investigations have shown that the magnetoelectric effect in the bulk laminate consisted of R-Fe alloy and ferroelectric oxide (e.g., $\mathrm{Tb}_{0.30} \mathrm{Dy}_{0.70} \mathrm{Fe}_{2}$ (Terfenol-D)/PZT) with magnetoelectric coupling coefficient $\alpha_{E}$ of $\sim 4680 \mathrm{mV} / \mathrm{cm}$.Oe is much larger than that of the all-oxide laminates (e.g., $\mathrm{CoFe}_{2} \mathrm{O}_{4} / \mathrm{PZT}$; its $\alpha_{E}$ is $\sim 60 \mathrm{mV} / \mathrm{cm} \cdot \mathrm{Oe}$ ) [23-25]. However, with the applications in the micro-electro-mechanical system (MEMS) devices such as microtransducers, microactuators, 


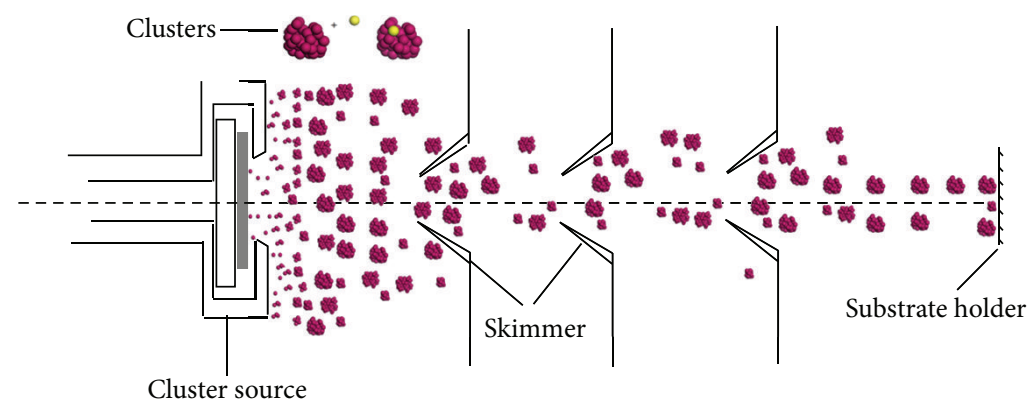

FIgURE 1: The sketched diagram of the growth and deposition process of clusters.

and microsensors [26], the well-defined microstructures together with the tunable properties are necessary for the multiferroics. Therefore, for the laminated composite film (i.e., thin-film heterostructure), it could be expected that magnetoelectric effect would be enhanced significantly if the $\mathrm{R}-\mathrm{Fe}$ alloy is used in the magnetostriction layer. Though the giant magnetostrictive films have been prepared by song conventional film preparation means [27-30], since the phase-formation temperature of R-Fe alloy is very high (the substrate is generally heated above $500^{\circ} \mathrm{C}$ ), it is unavoidable to bring about serious oxygen diffusion from PZT oxide to $\mathrm{Tb}$-Fe alloy. As a result, both magnetostriction in $\mathrm{Tb}-\mathrm{Fe}$ alloy and piezoelectricity in PZT are seriously suppressed. Moreover, the serious oxygen diffusion would also generate a new interface layer, which further significantly decreases the magnetoelectric coupling efficiency [31]. Therefore, a progress with low temperature and energy is necessary during deposition. Fortunately, we have developed an effective preparation method, namely, low energy cluster beam deposition (LECBD), to prepare the nanostructured magnetic, giant magnetostrictive, single phase multiferroic, and welldefined microstructured multiferroic heterostructured films [32-38]. This paper aims to review the breakthroughs on the multiferroic nanostructure assembled with clusters.

\section{Low Energy Cluster Beam Deposition}

As a very important building block of nanomaterials, nanoclusters are aggregates of atoms or molecules of nanometric size, containing a number of constituent particles ranging from 10 to $10^{6}$ [39-41]. All the beam experiments on clusters have a cluster source, in which the clusters are produced. There are a variety of sources available including arc cluster ion source [42], laser vaporization cluster source [43, 44], gas aggregation source $[45,46]$, seeded supersonic nozzle source [47], ion sputtering source $[48,49]$, and liquid metal ion source $[50,51]$. Based on these we developed low energy cluster beam deposition method. A magnetron-sputtering-gasaggregation (MSGA) cluster source produce is used to produce cluster beam to assemble multiferroic films. The growth and deposition process of clusters is shown in Figure 1 using gas aggregation. A direct current (DC) pulse power was used as the sputtering power. As the sputtering gas, one stream of argon gas with $99.9 \%$ purity was introduced through a ring structure close to the surface of the target. Another stream of argon was fed as a buffer gas through a gas inlet near the magnetron discharge head. The cluster condensation and growth region was cooled with liquid nitrogen. A highly oriented cluster beam with a small divergent angle less than one degree was formed by differential pumping controlled by the skimmers. During the process of the deposition, the average velocity perpendicular to the substrate of each cluster is quite low (with less than $50 \mathrm{meV} /$ atom corresponding to kinetic energy), and the velocity parallel to the substrate is about several meV/atom, which are both much lower than the molecular binding energy. The clusters are deposited on the substrate by a soft-landing manner and accumulated randomly but they do not coalesce with each other.

Based on this technique, various cluster-assembled nanostructured films such as metal and oxide have been prepared, which show peculiar properties different from the films prepared by the common methods [52-55]. Since the size, mass, and the assembling manner of the clusters can be precisely tuned by changing the working gas flow, controlling the length of condensation region, changing the buffer gas, and so forth, it is possible to control the microstructures and properties of the cluster-assembled nanostructured films, which makes it an ideal candidate for the fabrication of single phase or heterostructured films.

\section{Magnetic Films Assembled with Clusters}

3.1. Giant Magnetostrictive R-Fe Films. The cubic Laves phase $\mathrm{R}-\mathrm{Fe}(\mathrm{R}=$ rare earth element such as $\mathrm{Tb}, \mathrm{Dy}, \mathrm{Sm})$ compounds are well known to be a giant magnetostrictive material at room temperature, which could be widely used as actuators, transducers, dampers, and so forth [56, 57]. With the demand of the rapidly developing nano-electromechanical system (NEMS), much work has been done and various methods such as ion plating [27], ion beam sputtering [28], flash evaporation [29] and magnetron sputtering [58], and molecular beam epitaxy $[59,60]$ have been developed for the preparation of R-Fe films. However, compared with the bulk materials, the saturation magnetostriction of the current R-Fe films is much lower while the magnetic driving field is still higher [58], which limits them to be further used. Fortunately, based on the LECBD technique, a welldefined $\mathrm{Tb}$-Fe nanostructured film has been obtained, which 

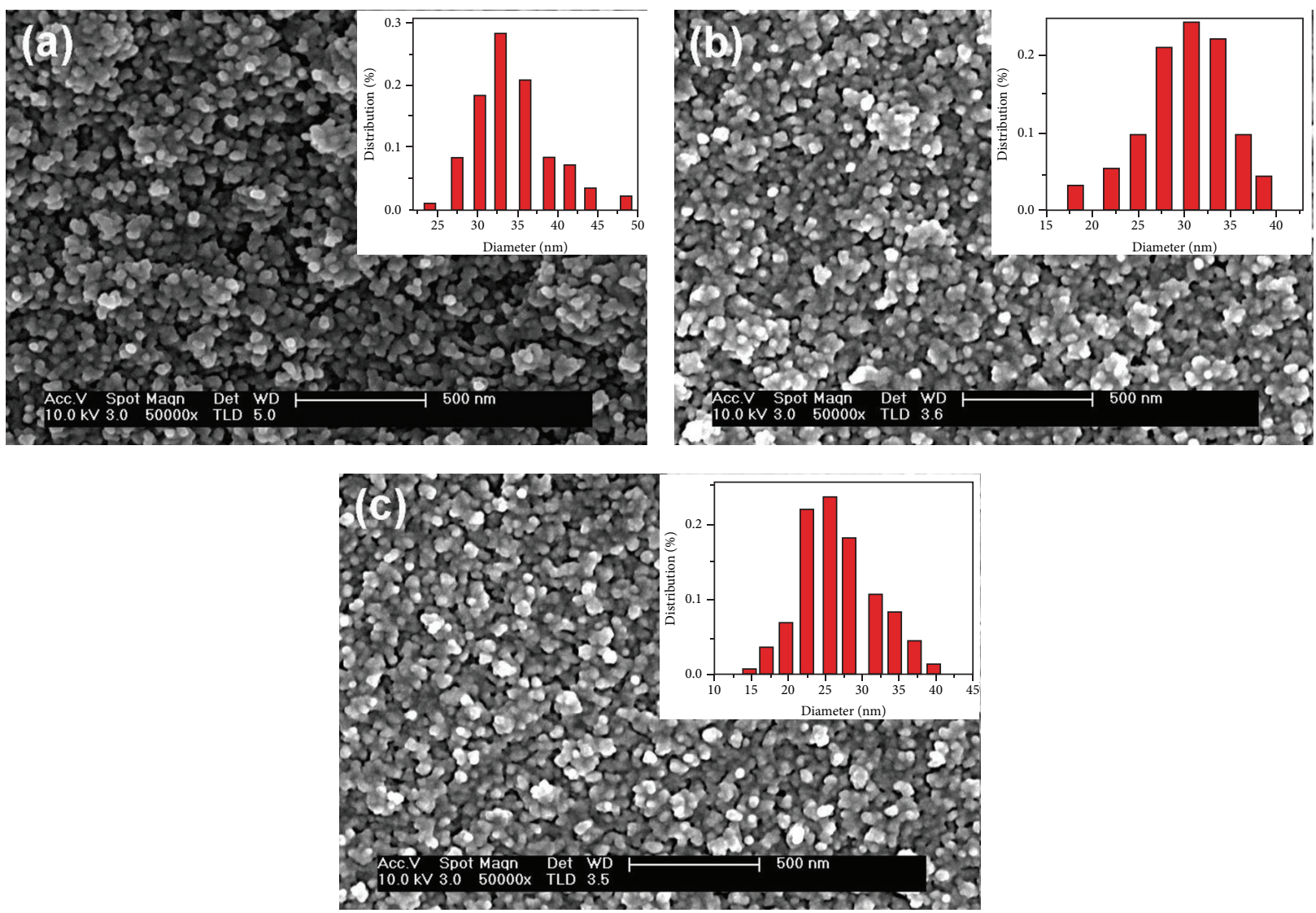

FIGURE 2: SEM images of the typical as-deposited Tb-Fe films and the graph of population versus size distribution of the nanoparticles at different growth region length. (a) $110 \mathrm{~mm}$, (b) $950 \mathrm{~mm}$ (c), and $80 \mathrm{~mm}$ ([36]).

exhibits excellent magnetostriction, much higher than the common Tb-Fe films [34]. Since the size, mass, and the assembling manner of the R-Fe clusters can be precisely tuned by changing the working gas flow, controlling the length of condensation region, changing the buffer gas, and so forth, it is possible to control the microstructures and magnetic properties of the cluster-assembled R-Fe nanostructured films, which makes it an ideal candidate for the fabrication of the magnetic NEMS devices.

A DC-magnetron-sputtering-gas-aggregation (MSGA) cluster source was used to produce the Tb-Fe cluster beam, which was finally deposited on the $\mathrm{Si}(100)$ substrate at room temperature to form the nanofilm. In order to tune the cluster size, the length of the condensing growth region $L$ was set as $80 \mathrm{~mm}, 95 \mathrm{~mm}$, and $110 \mathrm{~mm}$, respectively. Figure 2 presents the SEM images and size distributions of the typical TbFe films prepared under different length of the condensing growth region $L$. One can clearly observe two facts: (i) all films are assembled by the spherical nanoparticles, which are distributed uniformly and monodispersely in the film; (ii) with the increase of the condensing growth region length, the size of the nanoparticle increases, being in the ranges of $31 \sim 36 \mathrm{~nm}$ for $L=110 \mathrm{~mm}, 28 \sim 33 \mathrm{~nm}$ for $L=95 \mathrm{~mm}$, and $23 \sim 28 \mathrm{~nm}$ for $L=80 \mathrm{~mm}$, respectively. Meanwhile, we note that the particle size distribution was almost lognormal. The formation of such nanoparticle film is attributed to the unique LECBD preparation process. And the length of the cluster growth region significantly influences the size of the $\mathrm{Tb}-\mathrm{Fe}$ clusters for the films. In fact, the growth of the clusters as well as their size distribution is mainly determined by the cluster residence time and its distribution $[61,62]$. With increasing the length of the condensing growth region, the cluster residence time in the condensing growth region increases, and thus the collision among metal ion, Tb-Fe vapor, carrier gas, and free clusters becomes more sufficient, leading to the bigger size of the clusters.

It has been confirmed that all present nanoparticleassembled Tb-Fe films exhibit higher magnetostriction comparing to the common nonnanostructured films prepared by other methods [63-65]. And we observe that the magnetostrictive behavior and piezomagnetic coefficient evidently vary with the average size of the nanoparticle. Figure 3 gives the magnetostriction and piezomagnetic coefficient on the magnetic field for the films with various particle sizes. With increasing the particle size, the saturation magnetostriction $\lambda_{s}$ and the saturation magnetic field $H_{m}$ change, for example, $\lambda_{s} \sim 816 \times 10^{-6}$ and $H_{m} \sim 6.0 \mathrm{kOe}$ for $d=25 \mathrm{~nm}, \lambda_{s} \sim 1029 \times$ $10^{-6}$ and $H_{m} \sim 7.0 \mathrm{kOe}$ for $d=30 \mathrm{~nm}$, and $\lambda_{s}=746 \times$ $10^{-6}$ and $H_{m}=5.0 \mathrm{kOe}$ for $d=35 \mathrm{~nm}$. Obviously, the film with $d=30 \mathrm{~nm}$ has the highest saturation magnetostriction and piezomagnetic coefficient. However, it is not the case at low magnetic field. The film with $d=35 \mathrm{~nm}$ possesses 


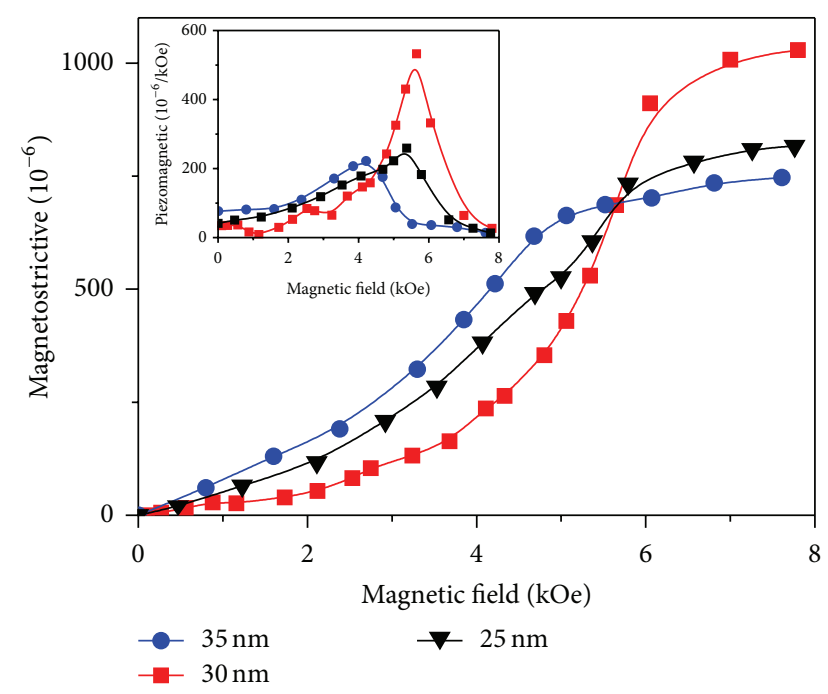

FIgURE 3: The dependence of magnetostriction and piezomagnetic coefficient for the $\mathrm{Tb}-\mathrm{Fe}$ nanostructured film on various particle sizes.

higher magnetostriction and piezomagnetic coefficient at low magnetic field.

It suggests that the dependence of the magnetostriction and piezomagnetic coefficient on the particle size could be attributed to the difference of the magnetization characteristic for these films. Figure 4 presents the field dependent magnetization at room temperature for the films with the particle sizes of $25 \mathrm{~nm}, 30 \mathrm{~nm}$, and $35 \mathrm{~nm}$. It is shown that the degree of magnetization anisotropy for the film is significantly affected by the particles size. Both in-plane and out-of-plane saturation magnetization change, as well as the coercivity with variation of the particle size. For the films with particles size of $30 \mathrm{~nm}$, the degree of magnetic anisotropy is the maximum and the difference between in-plane and outof-plane saturation magnetization is maximum. The easy axis is out-of-plane. Thus particle size dependence of the magnetic anisotropy for the present films should be correlative to the exchange coupling effects between the nanoparticles in the film [66]. Thus the film with $d=30 \mathrm{~nm}$ may show the highest degree of magnetic anisotropy because the exchange coupling distance is twice of the domain wall width $(\mathrm{RFe} \sim 15 \mathrm{~nm})$ for magnetic nanoparticles [67]. Therefore, for the film with $d=30 \mathrm{~nm}$, it has higher magnetic anisotropy than the other films. It needs far higher magnetic field to rotate the spin into the applied field. Therefore, the magnetostrictive coefficient of this film is lower than the other films at a low magnetic field, but, its saturation magnetostriction is the highest, which could be ascribed to the higher energy of anisotropy exchange interaction [68].

3.2. Enhanced Ferromagnetism of $\mathrm{BiFeO}_{3}$ Films Assembled with Clusters. $\mathrm{BiFeO}_{3}(\mathrm{BFO})$ is one of the most outstanding single-phase lead-free multiferroics due to its high ferroelectric Curie $\left(T_{\mathrm{FE}} \sim 1103 \mathrm{~K}\right)[69]$ and Neel $\left(T_{N} \sim 643 \mathrm{~K}\right)$ temperatures [70]. However, for BFO materials, antiferromagnetism and a superimposed incommensurate cycloid spin structure with a periodicity of $62 \mathrm{~nm}$ along the $\left[\begin{array}{lll}1 & 1 & 0\end{array}\right]_{h}$ axis cancel the macroscopic magnetization at room temperature, which restricts its applications [71]. Some investigations show that weak ferromagnetism is observed in some limited-dimension materials such as nanowires and nanoparticles due to the partial destruction of the spiral periodicity [72-74], which demonstrates a possible way to enhance ferromagnetism in single-phase multiferroics. Thus, as a size controllable building block of nanomaterials, clusters become a candidate to assemble multiferroics. Therefore, using LECBD technique, we have prepared the well-defined $\mathrm{BiFeO}_{3}$ nanostructured films assembled with 0 -characteristic-dimension clusters, and then the films were annealed at $600^{\circ} \mathrm{C}$. As we expected, the ferromagnetism of the as-prepared $\mathrm{BiFeO}_{3}$ films is enhanced [38].

Figure 5 gives the morphologies of cluster-assembled $\mathrm{BiFeO}_{3}$ nanostructured films before annealing. It can be seen that the films are assembled with clusters, which are nearly spherical and densely packed to form the uniformly continuous films, whereas each individual cluster is still clearly distinguishable. The population versus the size reveals that the average size of the nanoparticles is $\sim 22 \mathrm{~nm}$ for asdeposited films and $\sim 25.5 \mathrm{~nm}$ for the annealed films and is attributed to the fact that the size of cluster increases derive from the improvement of crystallizing during the annealing process.

Figure 6 present the XRD patterns of the typical asdeposited and annealed nanostructured films assembled with clusters. They show that both present films are polycrystalline and all of the observed diffraction peaks can be indexed to a perovskite structure. And the as-deposited BFO nanostructured films and other common films belonging to the rhombohedral structure with space group R-3c (161) prepared by other methods annealed BFO films transform to the coexistence of tetragonal and orthorhombic symmetry structure as (104) and (110) diffraction peaks are not obviously split, which is observed by expanding the view of the XRD pattern around $2 \theta=32.6^{\circ}$ in the inset of Figure 6 . At the same time, the lattice constant of cluster-assembled $\mathrm{BiFeO}_{3}$ films is $a=5.491 \AA$, smaller than those of the films prepared by other methods. It suggests that there exists a crystal distortion for the cluster-assembled BFO films, giving rise to a transition from the rhombohedral structure to tetragonal one [75-77], which means the crystal structure changes from a high symmetry state to a low symmetry state compared to bulk BFO materials. Thus the crystal distortion is due to the size effect of the clusters with the smaller characteristic size, which partially destroys the long-range cycloid spin structure with a periodicity of $62 \mathrm{~nm}$ in the rhombohedral structure with space group R-3c (161). It is such crystal distortion of the as-prepared films that brings about the enhancement in magnetization.

Figure 7 shows the magnetic hysteresis loops for the cluster-assembled BFO nanostructured films measured at $5 \mathrm{~K}$ and $300 \mathrm{~K}$. As can be seen, obvious ferromagnetism is observed for the cluster-assembled BFO nanostructured films not only at $5 \mathrm{~K}$ but also at room temperature. In Particular, the saturation magnetization of the BFO films at room temperature reaches $108 \mathrm{emu} / \mathrm{cc}$, which is comparable 


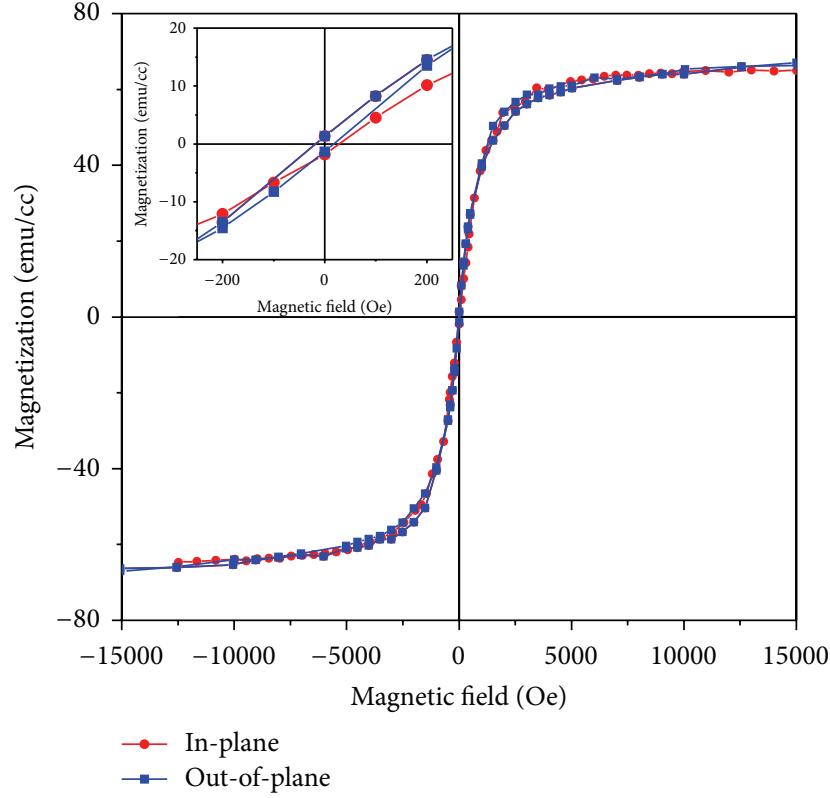

(a)

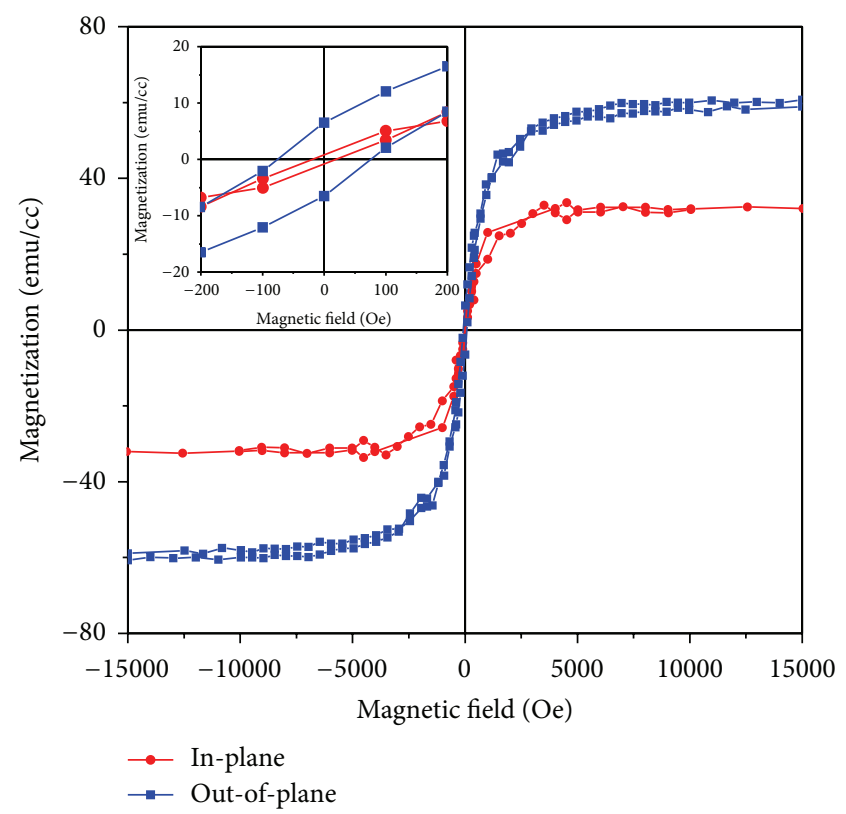

(b)

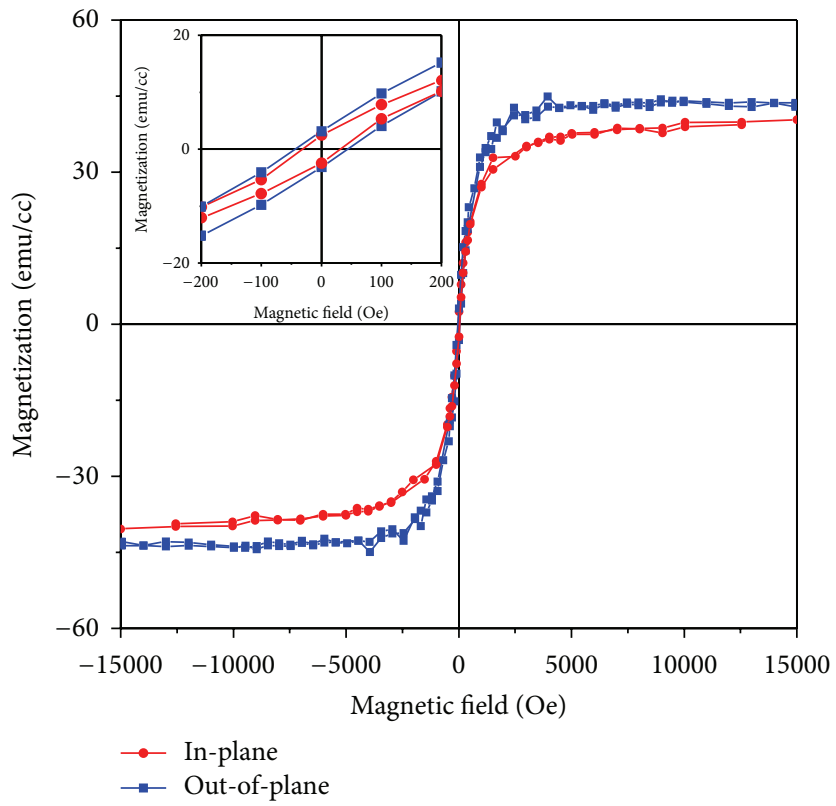

(c)

FIGURE 4: Magnetic hysteresis loops for the Tb-Fe nanostructured film assembled by the clusters (a) with $35 \mathrm{~nm}$ in diameter, (b) with $30 \mathrm{~nm}$ in diameter, and (c) with $25 \mathrm{~nm}$ in diameter.

with that of the present films with $125 \mathrm{emu} / \mathrm{cc}$ at $5 \mathrm{~K}$. More importantly, the large magnetization of $81 \mathrm{emu} / \mathrm{cc}$ is obtained at a magnetic field of 3000 Oe at room temperature, which is a larger response than the common films prepared by other methods [78-80].

Such enhanced room temperature ferromagnetism is attributed to the fact the average size of BFO clusters is much less than the long-range cycloid order of $62 \mathrm{~nm}$ along the $\left[\begin{array}{lll}1 & 1 & 0\end{array}\right]_{h}$ axis that the periodicity of the spin cycloid is broken [81]. Antiferromagnetic materials are considered as the combination of one sublattice with spins along one direction and another with spins along the opposite direction. If no spin canting is considered, the spins of these two sublattices compensate each other so that the net magnetization inside the material would become zero [82]. However, the long-range antiferromagnetic order is frequently interrupted at the cluster surfaces, which forms the uncompensated surface spins. For the cluster-assembled BFO films with the average size of $25.5 \mathrm{~nm}$, the uncompensated surface spins become very significant due to the very large surface to 

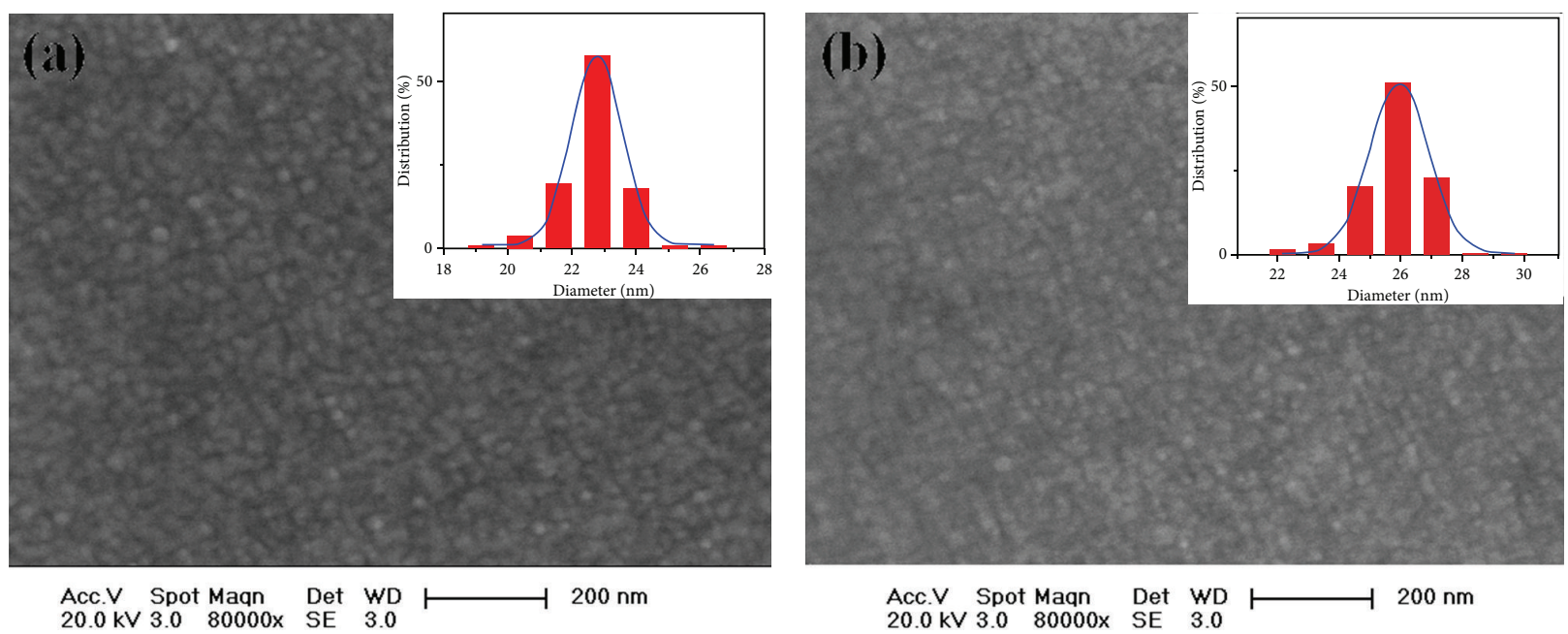

FIGURE 5: (a) Typical SEM image of as-deposited BFO nanostructured films, (b) the films after annealing, and the inset is the graph of population versus size distribution of the clusters.

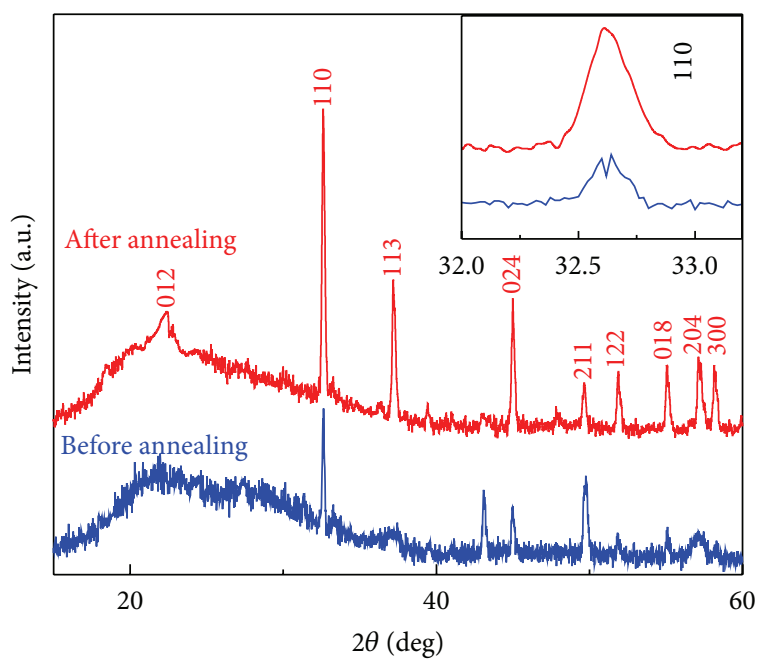

FIGURE 6: The XRD patterns before annealing and after annealing; the inset is the expanded view on the location of diffraction peak around $2 \theta=32.6^{\circ}$.

volume ratio for the clusters. The uncompensated spins at the surface enhance the contribution to the nanoparticle's overall magnetization. Besides, structural distortion and change of lattice parameter due to the size effect for the clusterassembled nanostructured films [83] lead to the release of the latent magnetization locked within the cycloid. Then the ferromagnetism of nanostructured films is significantly enhanced.

\section{Multiferroic Film Heterostructure Assembled with Clusters}

It is well known that composite films combined with piezoelectric and magnetostrictive materials can obtain stronger magnetoelectric effect than single phase materials [84] by the magnetic-mechanical-electric coupling product interaction via the stress mediation. Some composite films combined by perovskite ferroelectric oxides (e.g., $\mathrm{Pb}\left(\mathrm{Zr}_{0.52} \mathrm{Ti}_{0.48}\right) \mathrm{O}_{3}$ $(\mathrm{PZT}), \mathrm{BaTiO}_{3}$ ) with ferromagnetic oxides (e.g., $\mathrm{CoFe}_{2} \mathrm{O}_{4}$, $\left.\mathrm{La}_{0.67} \mathrm{Sr}_{0.33} \mathrm{MnO}_{3}\right)[18,21,85,86]$ did not acquire strong magnetoelectric effect due to low magnetostriction of the ferromagnetic oxides. Since we had prepared giant magnetostrictive R-Fe films using low energy cluster beam deposition, it is possible to prepare the well-defined microstructured thinfilm multiferroic heterostructure consisting of R-Fe alloy and ferroelectric oxide. And the substrate is ferroelectric oxide; the degree of the interfacial reaction or diffusion between $\mathrm{Tb}-\mathrm{Fe}$ alloy and ferroelectric oxide would be greatly suppressed due to the low temperature and energy during LECBD progress. Thus well-defined microstructure of thinfilm heterostructure as well as strong magnetoelectric effect would be obtained.

4.1. Tb-Fe/PZT Thin-Film Heterostructure. Tb-Fe nanocluster beam was deposited onto the surface of the PZT film through the open holes of the mask by LECBD progress. After deposition, not taking off the mask, a Pt electrode layer was deposited on the Tb-Fe dots via pulse laser deposition. Figure 8 presents the surface SEM image of the Tb-Fe layer in the heterostructure. It shows that the $\mathrm{Tb}$-Fe layer is compactly assembled by the regular spherical nanoclusters, which are distributed uniformly and adjacent with each other. The structure of the thin-film heterostructure is sketched in Insert (a) of Figure 8. Insert (b) of Figure 8 shows the cross-sectional SEM image of the thin-film heterostructure. One observes that the interface between Tb-Fe and PZT layers is clear and no transition layer is observed, which is benefit from the LECBD progress. During this process, the phase formation of $\mathrm{Tb}-\mathrm{Fe}$ nanoclusters (or nanoparticles) is achieved in the condensation chamber with high temperature, while the deposition of $\mathrm{Tb}-\mathrm{Fe}$ nanocluster beam onto the substrate is achieved in another high vacuum chamber with low energy 


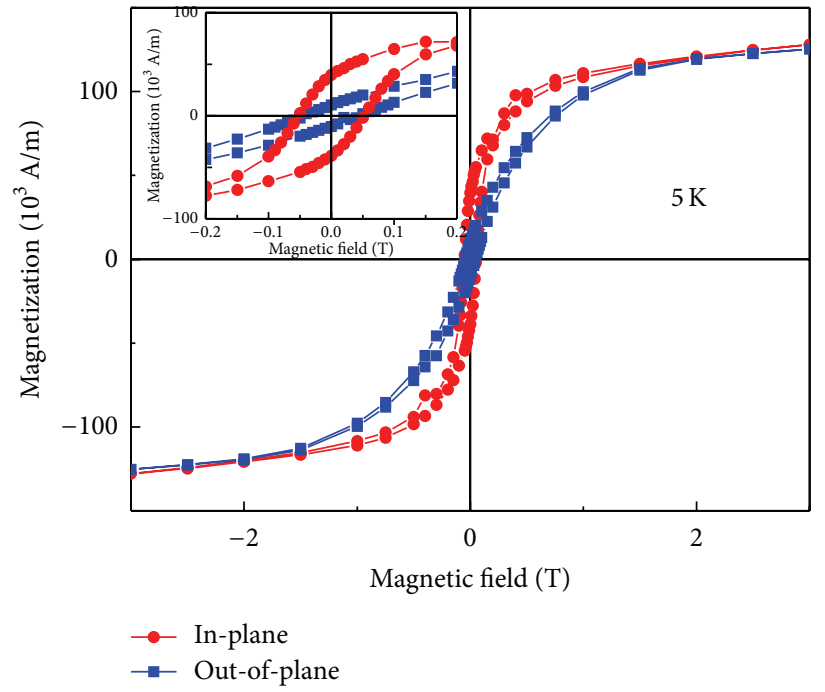

(a)

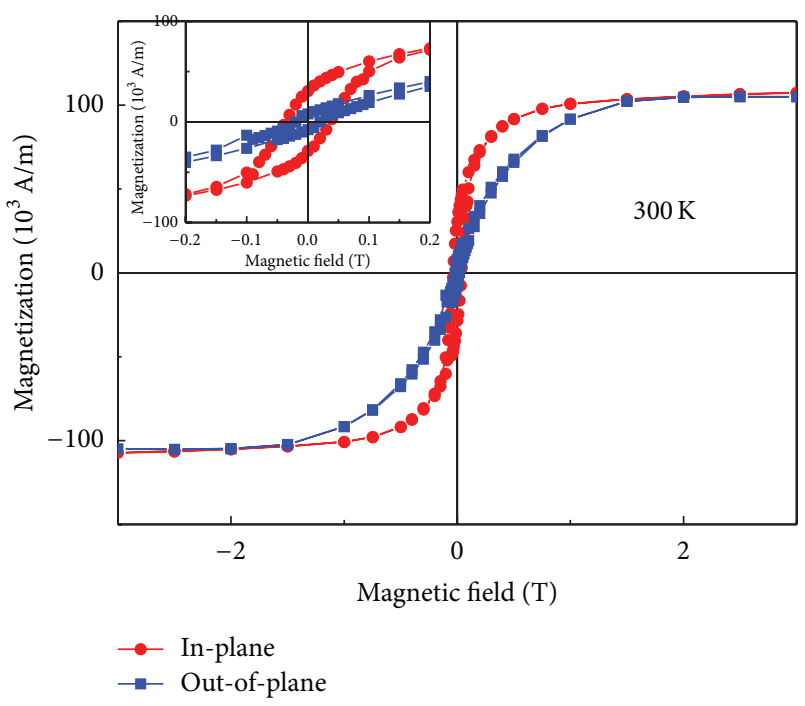

(b)

FIgURE 7: The magnetization dependence on the magnetic field of the typical cluster-assembled BFO nanostructured films (a) at low temperature of $5 \mathrm{~K}$, (b) at $300 \mathrm{~K}$. The inset is the expand view of the magnetic hysteresis.

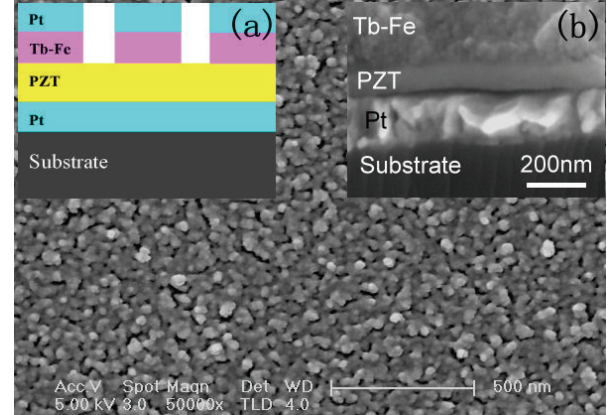

Figure 8: The surface SEM image of the Tb-Fe layer in the thinfilm heterostructure. Insert (a) is a sketch of the heterostructure, and Insert (b) is the typical cross-sectional SEM image of the heterostructure.

and low temperature (e.g., room temperature). Both processes are independent of each other. It is easy to understand no reaction between $\mathrm{Tb}-\mathrm{Fe}$ and $\mathrm{PZT}$ layers.

No destroying between $\mathrm{Tb}-\mathrm{Fe}$ and PZT layers gives a feasibility of well ferroelectric and ferromagnetic properties. Figure 9 gives the polarization versus electric field hysteresis loops and magnetic hysteresis loops for the Tb-Fe/PZT thinfilm heterostructure. It shows that the well-defined ferroelectric loops are observed. The saturation polarization and remanent polarization for $\mathrm{Tb}-\mathrm{Fe} / \mathrm{PZT}$ thin-film heterostructure have a very slight decrease compared with the pure PZT film. Such slight decrease in ferroelectric properties of the heterostructure should be attributed to the increase of oxygen vacancy concentration in PZT layer, which brings about difficulty for the mobility of domain walls in a certain degree and further leads to the decrease in polarization [87]. Insert of Figure 9(a) shows that the leakage current density in the heterostructure is quite low, for example, only being $\sim 1.5 \times$ $10^{-4} \mathrm{~A} / \mathrm{cm}^{2}$ even under the higher electric field of $30 \mathrm{MV} / \mathrm{m}$. In spite of this, we found that the leakage current density in the heterostructure was still higher than that of the pure PZT film, which indicates the increase of free carrier density in PZT layer of the heterostructure [88].

Besides, the heterostructure exhibits the well-defined magnetic hysteresis loops. It shows that both in-plane and out-of-plane coercive field are the same as only $H_{c} \sim$ $60 \mathrm{Oe}$, much lower than that of the bulk Tb-Fe alloy, while the in-plane and out-of-plane saturation magnetizations are, respectively, $\sim 38 \mathrm{emu} / \mathrm{cm}^{3}$ and $\sim 47 \mathrm{emu} / \mathrm{cm}^{3}$. We notice that the magnetization character of the heterostructure is almost comparable to the pure nanostructured Tb-Fe film prepared by LECBD process. Since magnetoelectric effect in a two-phase composite mainly originates from the interfacial stress transfer between the magnetostrictive and the ferroelectric phase, the high magnetostriction and ferroelectrics are beneficial to the magnetoelectric coupling. A strong magnetoelectric coupling could be obtained in the thin-film heterostructure.

Figure 10 plots the magnetic bias $H_{\text {bias }}$ dependence of the induced voltage increment $\left|\Delta V_{\mathrm{ME}}\right|$ at a given ac magnetic field frequency $f=1.0 \mathrm{kHz}$. It shows that thin-film heterostructure exhibits strong magnetoelectric coupling. The calculated maximum increment of the magnetoelectric voltage coefficient is as high as $\sim 140 \mathrm{mV} / \mathrm{cm} \cdot \mathrm{Oe}$, larger than that of the reported all-oxide ferroelectric-ferromagnetic composite film [16-18]. So strong magnetoelectric effect in $\mathrm{Tb}-\mathrm{Fe} / \mathrm{PZT}$ thin-film heterostructure is evidently beneficial from the unique LECBD process. Based on this process, not only could the interface reaction be availably avoided on the maximum degree, but also both ferroelectric and magnetostrictive properties for PZT and $\mathrm{Tb}-\mathrm{Fe}$ could be maintained well. 


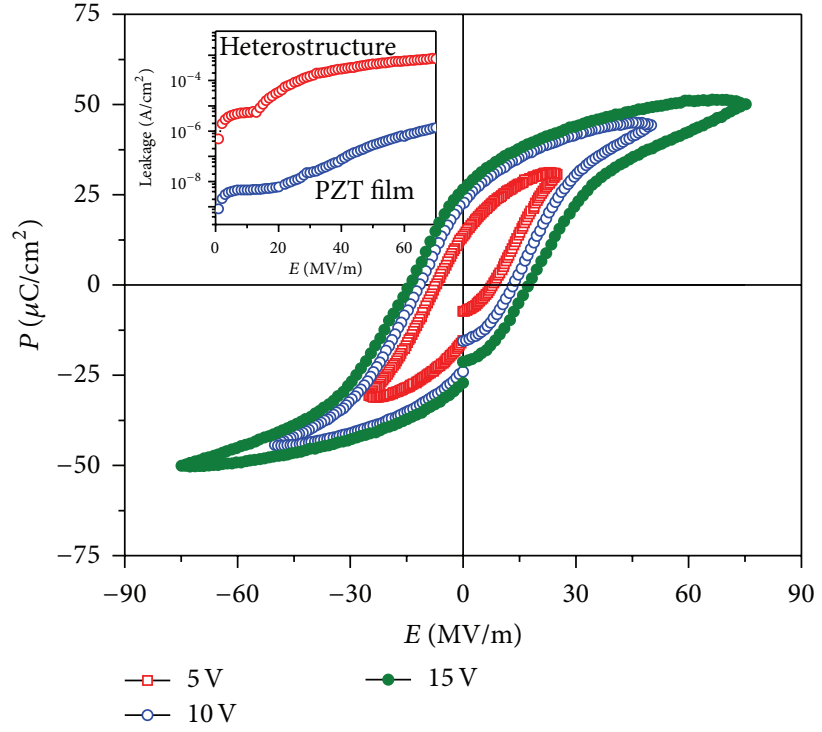

(a)

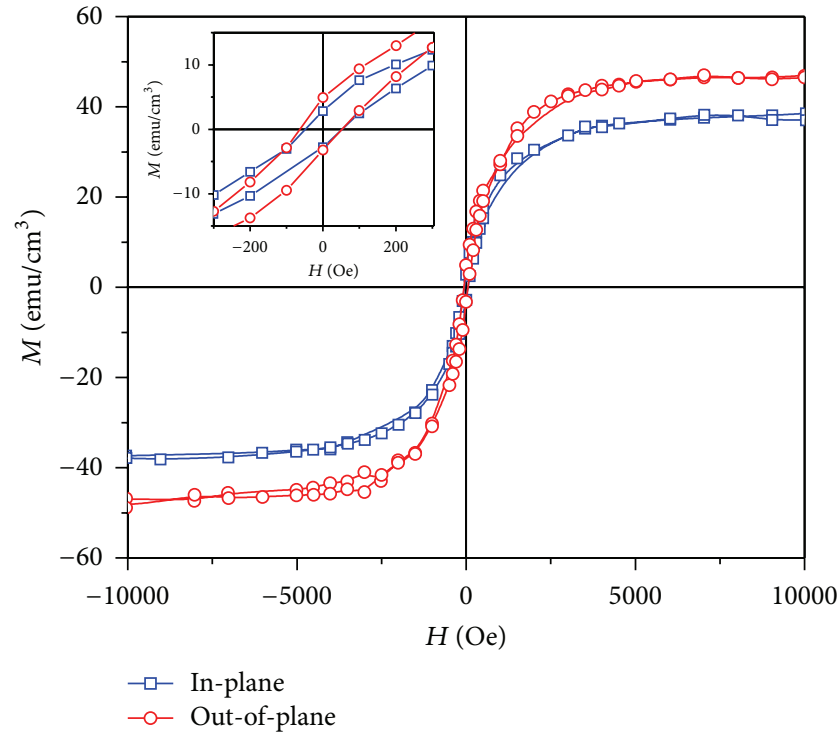

(b)

Figure 9: (a) Polarization versus electric field hysteresis $(P-E)$ loops for the thin-film heterostructure. Insert is the variation of leakage current density with the applied electric field. (b) The field dependent magnetization $(M-H)$ curves for the thin-film heterostructure.

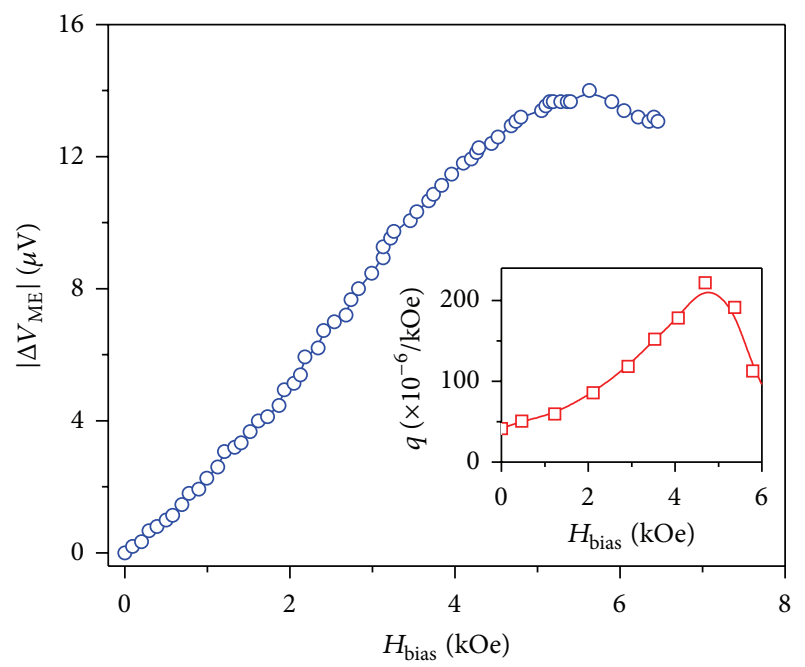

FIgURE 10: The $H_{\text {bias }}$ dependence of the induced magnetoelectric voltage increment $\left|\Delta V_{\mathrm{ME}}\right|$ at a given dc magnetic frequency $f=$ $1.0 \mathrm{kHz}$ for the thin-film heterostructure. Inset is the $H_{\text {bias }}$ dependence of piezomagnetic coefficient for the pure $\mathrm{Tb}$-Fe nanostructured film prepared by LECBD process.

Insert of Figure 10 shows the $H_{\text {bias }}$ dependence of piezomagnetic coefficient $q\left(=\delta \lambda / \delta H_{\text {bias }}\right)$ for the pure TbFe nanostructured film prepared by LECBD process. Both $\left|\Delta V_{\mathrm{ME}}\right|$ in heterostructure and $q$ in Tb-Fe film have the similar change trend with $H_{\text {bias }}$. This indicates that the magnetoelectric coupling in the heterostructure should be dominated by the magnetic-mechanical-electric transform through the stress-mediated transfer.
4.2. Sm-Fe/PVDF Thin-Film Heterostructure. For Si based magnetoelectric composited films, the couple efficiency between ferroelectric and ferromagnetic phases was depressed due to the stress clamping effect of the hard substrate. Therefore a flexible polyvinylidene fluoride (PVDF) film may be used instead of the hard substrate due to its small Young's modulus. Thus the magnetoelectric coupling between the ferroelectric and ferromagnetic phases will almost be not influenced. Moreover, piezoelectric voltage constant $\left(g_{31}\right)$ of PVDF film is an order higher than that of ordinary PZT film, which allows it to generate a bigger voltage output under a small stress. This indicates that PVDF film is suitable to act as the piezoelectric phase in the magnetoelectric thin-film heterostructure.

The flexible PVDF/Sm-Fe heterostructural film was prepared by depositing $\mathrm{Sm}$-Fe nanocluster beam onto the PVDF film at room temperature using LECBD technique. Though it is very easy to destroy the PVDF polymer substrate, it can be avoidable during LECBD progress with quite low energy and low temperature. Figure 11 shows the cross-section SEM image of the Sm-Fe/PVDF film. It can clearly be observed that the interface between the PVDF film and Sm-Fe layer is clear and no evident transition layer appears, indicating that the PVDF film does not get destroyed during the process of LECBD. The well-defined heterostructure makes it possible to generate the strong magnetoelectric effect.

The Sm-Fe film exhibits strong negative magnetostrictive effect with a saturation value of $\sim 750 \times 10^{-6}$ at magnetic field of $\sim 7.0 \mathrm{kOe}$ as shown in Figure 12. Inset of Figure 12 shows that the Sm-Fe/PVDF film exhibits distinct magnetic anisotropy with an in-plane magnetic easy axis, which obviously makes the magnetoelectric coupling in the Sm$\mathrm{Fe} / \mathrm{PVDF}$ film be more efficient under an in-plane magnetic field. 


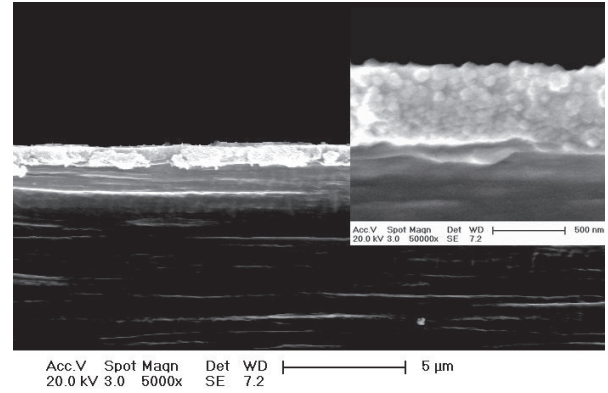

FIgURE 11: The cross-section SEM image of the Sm-Fe/PVDF thinfilm heterostructure.

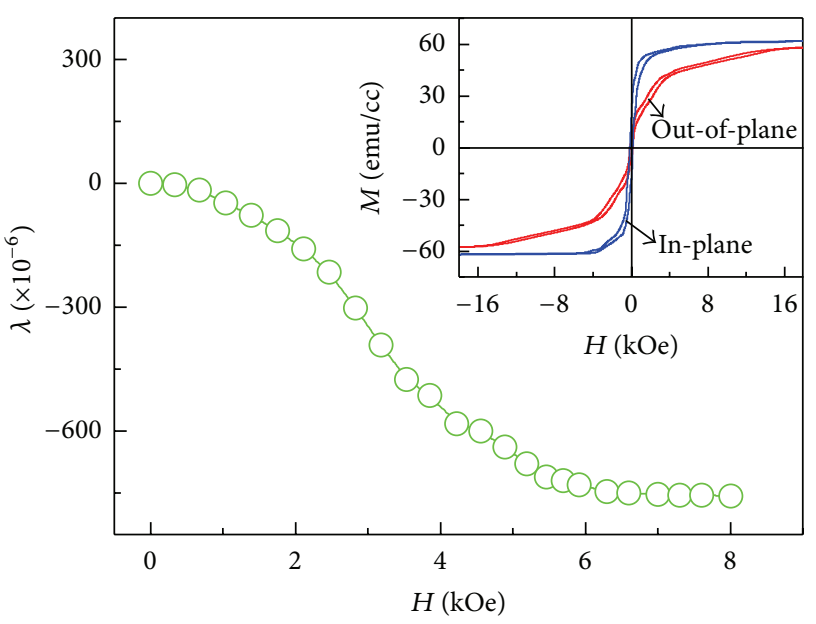

Figure 12: Magnetostriction $\lambda$ dependence of Sm-Fe film on magnetic field $H$. Inset is the magnetic hysteresis loops for the Sm$\mathrm{Fe} / \mathrm{PVDF}$ thin-film heterostructure measured at room temperature.

For thin-film heterostructure, a large magnetoelectric voltage output can be obtained. Figure 13 gives the magnetoelectric voltage output increment $\left|\Delta V_{\mathrm{ME}}\right|$ value as a function of $H_{\text {bias }}$ for the Sm-Fe/PVDF film. It is seen that the film exhibits a large voltage output under the external magnetic bias. The $\left|\Delta V_{\mathrm{ME}}\right|$ value increases with increasing $H_{\text {bias }}$, reaching the maximum value of $\left|\Delta V_{\mathrm{ME}}\right| \sim 210 \mu \mathrm{V}$ at $H_{\text {bias }}=2.3 \mathrm{kOe}$, and then drops. Compared with the previous investigations, the magnetoelectric voltage output in the present SmFe/PVDF film is remarkably large, almost being two orders higher than that of typical all-oxide $\mathrm{PZT} / \mathrm{CoFe}_{2} \mathrm{O}_{4} / \mathrm{PZT}$ film deposited on the hard wafer [89].

Therefore, by using the flexible PVDF polymer film as the substrate, the substrate clamping effect on the magnetoelectric coupling of the heterostructural film is completely eliminated. The heterostructural film exhibits large magnetoelectric voltage output, which is mainly attributed to the large piezoelectric voltage constant in the piezoelectric PVDF layer and high magnetic anisotropy with in-plane magnetic easy axis as well as the giant negative magnetostriction in the ferromagnetic Sm-Fe layer.

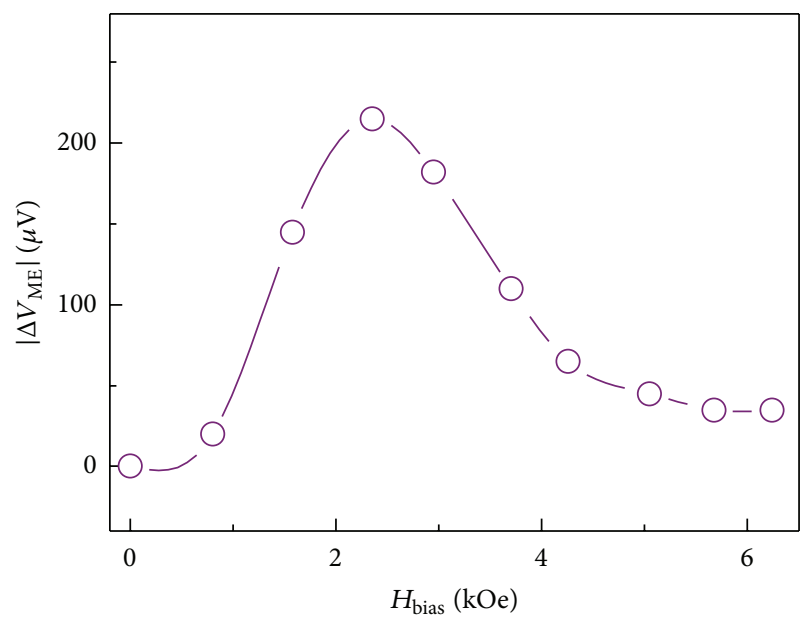

FIGURE 13: Induced voltage increment $\left|\Delta V_{\mathrm{ME}}\right|$ as a function of magnetic bias $H_{\text {bias }}$.

\section{Conclusions}

In a conclusion, the single phase multiferroic films and compound heterostructured multiferroic films assembled with clusters were prepared by low energy cluster beam deposition. It shows that the multiferroic properties of the thin-films can be controlled or improved by tuning the size of the clusters. And the structure of the thin-film heterostructure would not be destroyed due to low temperature and energy during LECBD progress. The LECBD technique provides an ideal avenue to prepare multiferroic nanostructure and facilitates their applications on NEMS devices.

\section{Conflict of Interests}

The author declares that there is no conflict of interests regarding the publication of this paper.

\section{Acknowledgments}

This work was financially supported by National Key Projects for Basic Research of China (973 Projects) (Grant no. 2012CB626815), the National Natural Science Foundation of China (Grant nos. 11264026, 10904065), the Program for Young Talents of Science and Technology in Universities of Inner Mongolia Autonomous Region (Grant no. NJYT-12B05), and Inner Mongolia Science Foundation for Distinguished Young Scholars (Grant no. 2014JQ01).

\section{References}

[1] J. Wang, J. B. Neaton, H. Zheng et al., "Epitaxial $\mathrm{BiFeO}_{3}$ multiferroic thin film heterostructures," Science, vol. 299, no. 5613, pp. 1719-1722, 2003.

[2] D. C. Arnold, K. S. Knight, F. D. Morrison, and P. Lightfoot, "Ferroelectric-paraelectric transition in : crystal structure of the orthorhombic phase," Physical Review Letters, vol. 102, Article ID 027602, 2009. 
[3] G. Giovannetti, S. Kumar, D. Khomskii, S. Picozzi, and J. van den Brink, "Multiferroicity in rare-earth nickelates $\mathrm{RNiO}_{3}$," Physical Review Letters, vol. 103, no. 15, Article ID 156401, 2009.

[4] C. Ederer and N. A. Spaldin, "Weak ferromagnetism and magnetoelectric coupling in bismuth ferrite," Physical Review B, vol. 71, Article ID 060401, 2005.

[5] W. Eerenstein, F. D. Morrison, J. Dho, M. G. Blamire, J. F. Scott, and N. D. Mathur, "Comment on "epitaxial $\mathrm{BiFeO}_{3}$ multiferroic thin film heterostructures'”, Science, vol. 307, no. 5713, p. 1203, 2005.

[6] W. Eerenstein, N. D. Mathur, and J. F. Scott, "Multiferroic and magnetoelectric materials," Nature, vol. 442, no. 7104, pp. 759$765,2006$.

[7] D. Lebeugle, A. Mougin, M. Viret, D. Colson, and L. Ranno, "Electric field switching of the magnetic anisotropy of a ferromagnetic layer exchange coupled to the multiferroic compound $\mathrm{BiFeO}_{3}$," Physical Review Letters, vol. 103, no. 25, Article ID 257601, 2009.

[8] D. Lee, S. M. Yang, T. H. Kim et al., "Multilevel data storage memory using deterministic polarization control," Advanced Materials, vol. 24, no. 3, pp. 402-406, 2012.

[9] G. A. Smolenskii, A. I. Agranovskaya, S. N. Popov, and V. A. Isupov, "Dielectric polarization and losses of some complex compounds," Soviet Physics Uspekhi, vol. 3, p. 1981, 1958.

[10] E. Ascher, H. Rieder, H. Schmid, and H. Stöessel, "Some properties of ferromagnetoelectric nickel-iodine boracite, $\mathrm{Ni}_{3} \mathrm{~B}_{7} \mathrm{O}_{13} \mathrm{I}$," Journal of Applied Physics, vol. 37, no. 3, pp. 1404-1405, 1966.

[11] D. L. Fox, D. R. Tilley, J. F. Scott, and H. J. Guggenheim, "Magnetoelectric phenomena in $\mathrm{BaMnF}_{4}$ and $\mathrm{BaMn}_{0.99} \mathrm{Co}_{0.01} \mathrm{~F}_{4}$," Physical Review B, vol. 21, no. 7, pp. 2926-2936, 1980.

[12] M. DiDomenico Jr., M. Eibschütz, H. J. Guggenheim, and I. Camlibel, "Dielectric behavior of ferroelectric $\mathrm{BaMF}_{4}$ above room temperature," Solid State Communications, vol. 7, no. 16, pp. 1119-1122, 1969.

[13] K. Kato, S. Iida, K. Yanai, and K. Mizushima, "Ferrimagnetic ferroelectricity of $\mathrm{Fe}_{3} \mathrm{O}_{4}$," Journal of Magnetism and Magnetic Materials, vol. 31-34, part 2, pp. 783-784, 1983.

[14] I. G. Ismailza and S. A. Kizhaev, "Determination of the Curie point of ferroelectrics $\mathrm{YMnO}_{3}$ and $\mathrm{YbMnO}_{3}$," Soviet Physics, Solid State, vol. 7, p. 236, 1965.

[15] J. R. Teague, R. Gerson, and W. J. James, "Dielectric hysteresis in single crystal $\mathrm{BiFeO}_{3}$," Solid State Communications, vol. 8, no. 13, pp. 1073-1074, 1970.

[16] H. Ryu, P. Murugavel, J. H. Lee et al., "Magnetoelectric effects of nanoparticulate $\mathrm{Pb}\left(\mathrm{Zr}_{0.52} \mathrm{Ti}_{0.48}\right) \mathrm{O}_{3}-\mathrm{NiFe}_{2} \mathrm{O}_{4}$ composite films," Applied Physics Letters, vol. 89, no. 10, Article ID 102907, 2006.

[17] Y. G. Ma, W. N. Cheng, M. Ning, and C. K. Ong, "Magnetoelectric effect in epitaxial $\mathrm{Pb}\left(\mathrm{Zr}_{0.52} \mathrm{Ti}_{0.48}\right) \mathrm{O}_{3} / \mathrm{La}_{0.7} \mathrm{Sr}_{0.3} \mathrm{MnO}_{3}$ composite thin film," Applied Physics Letters, vol. 90, Article ID 152911, 2007.

[18] J. P. Zhou, H. C. He, Z. Shi, and C. W. Nan, "Magnetoelectric $\mathrm{CoFe}_{2} \mathrm{O}_{4} / \mathrm{Pb}\left(\mathrm{Zr}_{0.52} \mathrm{Ti}_{0.48}\right) \mathrm{O}_{3}$ double-layer thin film prepared by pulsed-laser deposition," Applied Physics Letters, vol. 88, Article ID 013111, 2006.

[19] K. S. Chang, M. A. Aronova, C. L. Lin et al., "Exploration of artificial multiferroic thin-film heterostructures using composition spreads," Applied Physics Letters, vol. 84, no. 16, pp. 3091-3093, 2004.

[20] H. Zheng, J. Wang, S. E. Lofland et al., "Multiferroic $\mathrm{BaTiO}_{3}$ $\mathrm{CoFe}_{2} \mathrm{O}_{4}$ Nanostructures," Science, vol. 303, no. 5658, pp. 661663, 2004.
[21] W. Eerenstein, M. Wiora, J. L. Prieto, J. F. Scott, and N. D. Mathur, "Giant sharp and persistent converse magnetoelectric effects in multiferroic epitaxial heterostructures," Nature Materials, vol. 6, no. 5, pp. 348-351, 2007.

[22] R. C. Ohandley, Modern Magnetic Materials: Principles and Applications, John Wiley \& Sons, New York, NY, USA, 2000.

[23] J. Ryu, A. V. Carazo, K. Uchino, and H.-E. Kim, "Magnetoelectric properties in piezoelectric and magnetostrictive laminate composites," Japanese Journal of Applied Physics, vol. 40, no. 8, part 1, pp. 4948-4951, 2001.

[24] C. W. Nan, G. Liu, and Y. H. Lin, "Influence of interfacial bonding on giant magnetoelectric response of multiferroic laminated composites of $\mathrm{Tb}_{1-x} \mathrm{Dy}_{x} \mathrm{Fe}_{2}$ and $\mathrm{PbZr}_{x} \mathrm{Ti}_{1-x} \mathrm{O}_{3}$," Applied Physics Letters, vol. 83, p. 4366, 2003.

[25] G. Srinivasan, E. T. Rasmussen, A. A. Bush, K. E. Kamentsev, V. F. Meshcheryakov, and Y. K. Fetisov, "Structural and magnetoelectric properties of $\mathrm{MFe}_{2} \mathrm{O}_{4}$-PZT $(\mathrm{M}=\mathrm{Ni}, \mathrm{Co})$ and $\mathrm{La}_{x}(\mathrm{Ca}$, $\mathrm{Sr})_{1-x} \mathrm{MnO}_{3}$-PZT multilayer composites," Applied Physics A, vol. 78, no. 5, pp. 721-728, 2004.

[26] N. A. Spaldin and M. Fiebig, "The renaissance of magnetoelectric multiferroics," Science, vol. 309, no. 5733, pp. 391-392, 2005.

[27] H. H. Uchida, V. Koeninger, M. Wada et al., "Preparation and characterization of ( $\mathrm{Tb}, \mathrm{Dy}) \mathrm{Fe}_{2}$ giant magnetostrictive thin films for surface acoustic wave devices," Journal of Alloys and Compounds, vol. 211-212, pp. 455-459, 1994.

[28] M. Wada, H.-H. Uchida, Y. Matsumura, and H. Kaneko, "Preparation of films of (Tb,Dy) $\mathrm{Fe}_{2}$ giant magnetostrictive alloy by ion beam sputtering process and their characterization," Thin Solid Films, vol. 281-282, no. 1-2, pp. 503-506, 1996.

[29] H. Uchida, M. Wada, K. Koike et al., "Giant magnetostrictive materials: thin film formation and application to magnetic surface acoustic wave devices," Journal of Alloys and Compounds, vol. 211-212, pp. 576-580, 1994.

[30] E. Quandt, B. Gerlach, and K. Seemann, "Preparation and applications of magnetostrictive thin films," Journal of Applied Physics, vol. 76, no. 10, pp. 7000-7002, 1994.

[31] C. W. Nan, G. Liu, Y. H. Lin, and H. Chen, "Magnetic-fieldinduced electric polarization in multiferroic nanostructures," Physical Review Letters, vol. 94, no. 19, Article ID 197203, 4 pages, 2005.

[32] S. Zhao, J.-G. Wan, M. Yao, J.-M. Liu, F. Song, and G. Wang, "Flexible Sm-Fe/polyvinylidene fluoride heterostructural film with large magnetoelectric voltage output," Applied Physics Letters, vol. 97, no. 21, Article ID 212902, 2010.

[33] S. Zhao, Y. Wu, J.-G. Wan, X. Dong, J.-M. Liu, and G. Wang, "Strong magnetoelectric coupling in $\mathrm{Tb}-\mathrm{FePb}\left(\mathrm{Zr}_{0.52} \mathrm{Ti}_{0.48}\right) \mathrm{O}_{3}$ thin-film heterostructure prepared by low energy cluster beam deposition," Applied Physics Letters, vol. 92, no. 1, Article ID 012920, 2008.

[34] S. Zhao, F. Bi, J.-G. Wan et al., "Cluster-assembled Tb-Fe nanostructured films produced by low energy cluster beam deposition," Nanotechnology, vol. 18, no. 26, Article ID 265705, 2007.

[35] S. Zhao, M. Yao, J.-G. Wan, Y. Mu, J. Zhou, and G. Wang, "The microstructure and magnetic behavior of Co nanostructured film prepared by energetic cluster beam deposition," European Physical of Journal D, vol. 52, pp. 163-166, 2009.

[36] S. Zhao, J.-G. Wan, C. Huang et al., "The influence of nanoparticle size on the magnetostrictive properties of cluster-assembled Tb-Fe nanofilms," Thin Solid Films, vol. 518, no. 12, pp. 31903193, 2010. 
[37] S.-F. Zhao, C.-H. Yao, Q. Lu, F.-Q. Song, J.-G. Wan, and G.-H. Wang, "Cluster-assembled cobalt doped $\mathrm{ZnO}$ nanostructured film prepared by low energy cluster beam deposition," Transactions of Nonferrous Metals Society of China, vol. 19, no. 6, pp. 1450-1453, 2009.

[38] S. Zhao, Z. Ma, W. Xing et al., "Enhanced ferromagnetism of cluster-assembled $\mathrm{BiFeO}_{3}$ nanostructured films," Thin Solid Films, 2014.

[39] A. W. Castleman and K. H. Bowen, "Clusters: structure, energetics, and dynamics of intermediate states of matter," The Journal of Physical Chemistry, vol. 100, no. 31, pp. 12911-12944, 1996.

[40] R. L. Johnston, Atomic and Molecular Clusters, Taylor \& Francis, London, UK, 2002.

[41] D. J. Wales, Energy Landscapes: Applications to Clusters, Biomolecules and Glasses, Cambridge University Press, Cambridge, UK, 2004.

[42] R. P. Methling, V. Senz, E. D. Klinkenberg et al., "Magnetic studies on mass-selected iron particles," The European Physical Journal D, vol. 16, no. 1, pp. 173-176, 2001.

[43] V. E. Bondybey and J. H. English, "Laser induced fluorescence of metal clusters produced by laser vaporization: gas phase spectrum of $\mathrm{Pb}_{2}$," The Journal of Chemical Physics, vol. 74, no. 12, pp. 6978-6979, 1981.

[44] A. N. Andriotis and M. Menon, "Orbital magnetism: pros and cons for enhancing the cluster magnetism," Physical Review Letters, vol. 93, no. 2, Article ID 026402, 2004.

[45] J. Mühlbach, P. Pfau, E. Recknagel, and K. Sattler, "Cluster emission from the surfaces of Bi, Sb and Se," Surface Science, vol. 106, pp. 18-26, 1981.

[46] S. H. Baker, S. C. Thornton, K. W. Edmonds, M. J. Maher, C. Norris, and C. Binns, "The construction of a gas aggregation source for the preparation of size-selected nanoscale transition metal clusters," Review of Scientific Instruments, vol. 71, no. 8, pp. 3178-3183, 2000.

[47] W. A. de Heer, K. Selby, V. Kresin et al., "Collective dipole oscillations in small sodium clusters," Physical Review Letters, vol. 59, no. 16, pp. 1805-1808, 1987.

[48] T. Bergmann and T. P. Martin, "High-resolution time-of-flight mass spectrometers: part I. Effects of field distortions in the vicinity of wire meshes," Review of Scientific Instruments, vol. 60, no. 3, p. $347,1989$.

[49] T. Bergmann, T. P. Martin, and H. Schaber, "High-resolution time-of-flight mass spectrometer," Review of Scientific Instruments, vol. 60, no. 4, p. 792, 1989.

[50] N. D. Bhaskar, R. P. Frueholz, C. M. Klimcak, and R. A. Cook, "Evidence of electronic shell structure in $\mathrm{Rb}_{\mathrm{N}}{ }^{+}(N=1-100)$ produced in a liquid-metal ion source," Physical Review B, vol. 36, p. 4418, 1987.

[51] Y. Saito, K. Minami, T. Ishida, and T. Noda, "Abundance of Na cluster ions ejected from a liquid metal ion source," Zeitschrift für Physik D Atoms, Molecules and Clusters, vol. 11, no. 1, pp. 8791, 1989.

[52] F. Q. Song, M. Han, M. D. Liu, B. Chen, J. G. Wan, and G. $\mathrm{H}$. Wang, "Experimental observation of nanojets formed by heating PbO-coated Pb clusters," Physical Review Letters, vol. 94, no. 9, Article ID 093401, 4 pages, 2005.

[53] M. Han, J. F. Zhou, F. Q. Song et al., "Silicon-riched-oxide cluster assembled nanostructures formed by low energy cluster beam deposition," The European Physical Journal D, vol. 24, no. 1-3, pp. 269-272, 2003.
[54] J. G. Wan, M. Han, J. F. Zhou, and G. H. Wang, "Nanofilms of Si clusters confined in $\mathrm{SiO}$ nanoparticles prepared by low energy cluster beam deposition," Physics Letters A, vol. 280, no. 5-6, pp. 357-360, 2001.

[55] J. Antony, X. B. Chen, J. Morrison, L. Bergman, and Y. Qiang, "ZnO nanoclusters: synthesis and photoluminescence," Applied Physics Letters, vol. 87, Article ID 241917, 2005.

[56] T. Honda, K. I. Arai, and M. Yamaguchi, "Fabrication of magnetostrictive actuators using rare-earth $(\mathrm{Tb}, \mathrm{Sm})$ - Fe thin films (invited)," Journal of Applied Physics, vol. 76, no. 10, pp. 6994-6999, 1994.

[57] E. Quandt and F. Claeyssen, "Magnetostrictive materials and actuators," in Proceedings of the ACTUATORS, Messe Bremen $\mathrm{GmbH}$, Bremen, Germany, June 2000.

[58] Y. Hayashi, T. Honda, K. I. Arai, K. Ishiyama, and M. Yamaguchi, "Dependence of magnetostriction of sputtered $\mathrm{Tb}$ Fe films on preparation conditions," IEEE Transactions on Magnetics, vol. 29, no. 6, pp. 3129-3131, 1993.

[59] V. Oderno, C. Dufour, K. Dumesnil, P. Mangin, and G. Marchal, "Epitaxial growth of (110) $\mathrm{DyFe}_{2}, \mathrm{TbFe}_{2}$ and $\mathrm{Dy}_{0.7} \mathrm{~Tb}_{0.3} \mathrm{Fe}_{2}$ thin films by molecular beam epitaxy," Journal of Crystal Growth, vol. 165, no. 1-2, pp. 175-178, 1996.

[60] A. Mougin, C. Dufour, K. Dumesnil, and P. Mangin, "Straininduced magnetic anisotropy in single-crystal $R \mathrm{Fe}_{2}$ (110) thin films $\left(R=\mathrm{Dy}\right.$, Er, Tb, $\left.\mathrm{Dy}_{0.7} \mathrm{~Tb}_{0.3}, \mathrm{Sm}, \mathrm{Y}\right)$," Physical Review B: Condensed Matter and Materials Physics, vol. 62, no. 14, pp. 9517-9531, 2000

[61] J. Söderlund, L. B. Kiss, G. A. Niklasson, and C. G. Granqvist, "Lognormal size distributions in particle growth processes without coagulation," Physical Review Letters, vol. 80, no. 11, pp. 2386-2388, 1998.

[62] L. B. Kiss, J. Söderlund, G. A. Niklasson, and C. G. Granqvist, "Real origin of lognormal size distributions of nanoparticles in vapor growth processes," Nanostructured Materials, vol. 12, no. 1, pp. 327-332, 1999.

[63] A. G. Jenner, J. P. Hayes, L. A. Stone, H. V. Snelling, and R. D. Greenough, "Pulsed laser deposition-an alternative route to the growth of magnetic thin films," Applied Surface Science, vol. 138-139, no. 1-4, pp. 408-412, 1999.

[64] S. M. Na, S. J. Suh, and S. H. Lim, "Fabrication condition effects on the magnetic and magnetostrictive properties of sputtered Tb-Fe thin films," Journal of Applied Physics, vol. 93, no. 10, pp. 8507-8509, 2003.

[65] T. Yamaki, M. Sekine, T. Haraki, H. Uchida, and Y. Matsumura, "Giant magnetostrictive thin film formation by plasma process," Surface and Coatings Technology, vol. 169-170, pp. 613-615, 2003.

[66] G. Herzer, "Grain size dependence of coercivity and permeability in nanocrystalline ferromagnets," IEEE Transactions on Magnetics, vol. 26, no. 5, pp. 1397-1402, 1990.

[67] L. H. Lewis, A. R. Moodenbaugh, and D. O. Welch, "Stress, strain and technical magnetic properties in 'exchange-spring' $\mathrm{Nd}_{2} \mathrm{Fe}_{14} \mathrm{~B}+\alpha$-Fe nanocomposite magnets," Journal of Physics $D$ : Applied Physics, vol. 34, no. 5, pp. 744-751, 2001.

[68] J. Huang, C. Prados, J. E. Evetts, and A. Hernando, "Giant magnetostriction of amorphous $\mathrm{Tb}_{x} \mathrm{Fe}_{1-x}(0.10<x<0.45)$ thin films and its correlation with perpendicular anisotropy," Physical Review B, vol. 51, no. 1, pp. 297-304, 1995.

[69] D. C. Arnold, K. S. Knight, F. D. Morrison, and P. Lightfoot, "Ferroelectric-paraelectric transition in $\mathrm{BiFeO}_{3}$ : crystal structure of the orthorhombic $\beta$ phase," Physical Review Letters, vol. 102, no. 2, Article ID 027602, 4 pages, 2009. 
[70] D. Lebeugle, A. Mougin, M. Viret, D. Colson, and L. Ranno, "Electric field switching of the magnetic anisotropy of a ferromagnetic layer exchange coupled to the multiferroic compound $\mathrm{BiFeO}_{3}$," Physical Review Letters, vol. 103, no. 25, Article ID 257601, 2009.

[71] D. Sando, A. Agbelele, D. Rahmedov et al., "Crafting the magnonic and spintronic response of $\mathrm{BiFeO}_{3}$ films by epitaxial strain," Nature Materials, vol. 12, no. 7, pp. 641-646, 2013.

[72] S. Zhao and Q. Yun, "Enhanced ferromagnetism of Ho, Mn codoped $\mathrm{BiFeO}_{3}$ nanoparticles," Integrated Ferroelectrics, vol. 141, no. 1, pp. 18-23, 2013.

[73] S. Roy and S. B. Majumder, "Recent advances in multiferroic thin films and composites," Journal of Alloys and Compounds, vol. 538, pp. 153-159, 2012.

[74] T.-J. Park, G. C. Papaefthymiou, A. J. Viescas, A. R. Moodenbaugh, and S. S. Wong, "Size-dependent magnetic properties of single-crystalline multiferroic $\mathrm{BiFeO}_{3}$ nanoparticles," Nano Letters, vol. 7, no. 3, pp. 766-772, 2007.

[75] Z. L. Hou, H. F. Zhou, L. B. Kong, H. B. Jin, X. Qi, and M. S. Cao, "Enhanced ferromagnetism and microwave absorption properties of $\mathrm{BiFeO}_{3}$ nanocrystals with Ho substitution," Materials Letters, vol. 84, pp. 110-113, 2012.

[76] Q. Zhang, X. Zhu, Y. Xu et al., "Effect of $\mathrm{La}^{3+}$ substitution on the phase transitions, microstructure and electrical properties of $\mathrm{Bi}_{1-x} \mathrm{La}_{x} \mathrm{FeO}_{3}$ ceramics," Journal of Alloys and Compounds, vol. 546, pp. 57-62, 2013.

[77] N. Panwar, I. Coondoo, A. Tomar, A. L. Kholkin, V. S. Puli, and R. S. Katiyar, "Nanoscale piezoresponse and magnetic studies of multiferroic Co and Pr co-substituted BFO thin films," Materials Research Bulletin, vol. 47, no. 12, pp. 4240-4245, 2012.

[78] J. G. Wu, D. Q. Xiao, and J. G. Zhu, "Effect of (Bi, La)(Fe, Zn)O thickness on the microstructure and multiferroic properties of $\mathrm{BiFeO}_{3}$ thin films," Journal of Applied Physics, vol. 112, Article ID 094109, 2012.

[79] X. W. Tang, J. M. Dai, X. B. Zhu, and Y. P. Sun, "In situ magnetic annealing effects on multiferroic $\mathrm{Mn}$-doped $\mathrm{BiFeO}_{3}$ thin films," Journal of Alloys and Compounds, vol. 552, pp. 186-189, 2013.

[80] Y. Wang, Q. H. Jiang, H. C. He, and C. W. Nan, "Multiferroic $\mathrm{BiFeO}_{3}$ thin films prepared via a simple sol-gel method," Applied Physics Letters, vol. 88, Article ID 142503, 2006.

[81] I. Sosnowska, T. P. Neumaier, and E. Steichele, "Spiral magnetic ordering in bismuth ferrite," Journal of Physics C: Solid State Physics, vol. 15, no. 23, article 020, pp. 4835-4846, 1982.

[82] G. S. Arya and N. S. Negi, "Effect of In and Mn co-doping on structural, magnetic and dielectric properties of $\mathrm{BiFeO}_{3}$ nanoparticles," Journal of Physics D: Applied Physics, vol. 46, no. 9, Article ID 095004, 2013.

[83] K. Chakrabarti, K. Das, B. Sarkar, S. Ghosh, and S. K. De, "Enhanced magnetic and dielectric properties of Eu and Co codoped $\mathrm{BiFeO}_{3}$ nanoparticles," Applied Physics Letters, vol. 101, no. 4, Article ID 042401, 2012.

[84] J. Ryu, S. Priya, K. Uchino, and H.-E. Kim, "Magnetoelectric effect in composites of magnetostrictive and piezoelectric materials," Journal of Electroceramics, vol. 8, no. 2, pp. 107-119, 2002.

[85] Y. G. Ma, W. N. Cheng, M. Ning, and C. K. Ong, "Magnetoelectric effect in epitaxial $\mathrm{Pb}\left(\mathrm{Zr}_{0.52} \mathrm{Ti}_{0.48}\right) \mathrm{O}_{3} / \mathrm{La}_{0.7} \mathrm{Sr}_{0.3} \mathrm{MnO}_{3}$ composite thin film," Applied Physics Letters, vol. 90, Article ID 152911, 2007.

[86] H. Zheng, J. Wang, L. Mohaddes-Ardabili et al., "Threedimensional heteroepitaxy in self-assembled $\mathrm{BaTiO}_{3}-\mathrm{CoFe}_{2} \mathrm{O}_{4}$ nanostructures," Applied Physics Letters, vol. 85, no. 11, pp. 20352037, 2004.
[87] J. F. Scott and M. Dawber, "Oxygen-vacancy ordering as a fatigue mechanism in perovskite ferroelectrics," Applied Physics Letters, vol. 76, no. 25, pp. 3801-3803, 2000.

[88] C. Wang, M. Takahashi, H. Fujino et al., "Leakage current of multiferroic $\left(\mathrm{Bi}_{0.6} \mathrm{~Tb}_{0.3} \mathrm{La}_{0.1}\right) \mathrm{FeO}_{3}$ thin films grown at various oxygen pressures by pulsed laser deposition and annealing effect," Journal of Applied Physics, vol. 99, no. 5, Article ID 054104, 2006.

[89] Y. J. Wu, J. G. Wan, J. M. Liu, and G. H. Wang, "Significant enhancement of magnetoelectric output in multiferroic heterostructural films modulated by electric polarization cycles," Applied Physics Letters, vol. 96, Article ID 152902, 2010. 

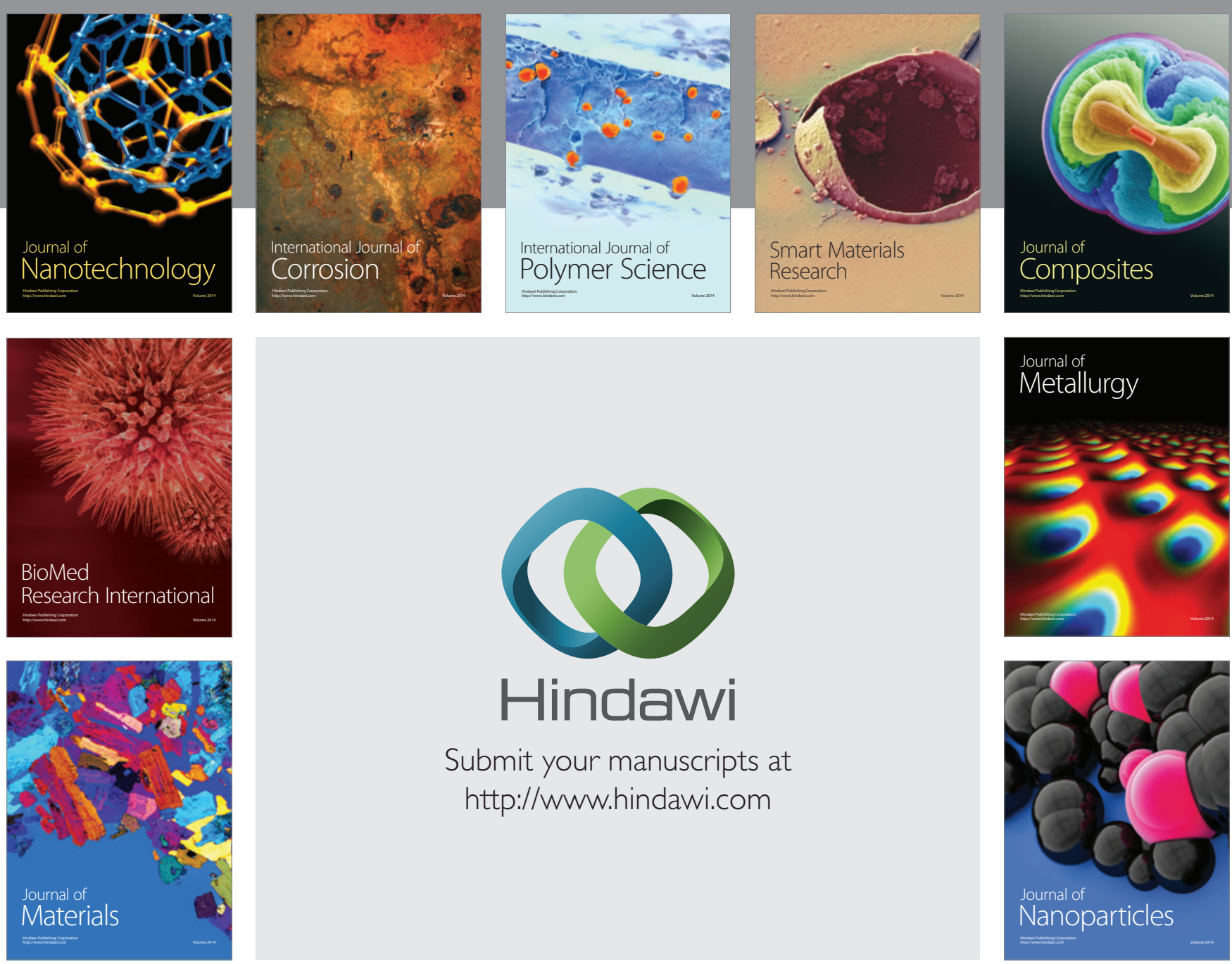

Submit your manuscripts at http://www.hindawi.com
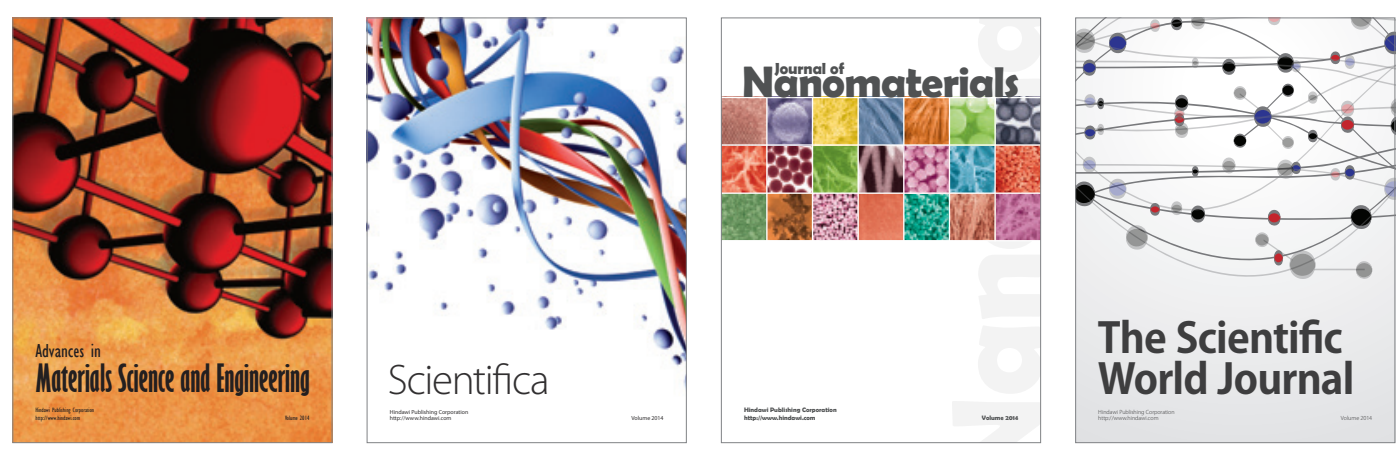

\section{The Scientific World Journal}
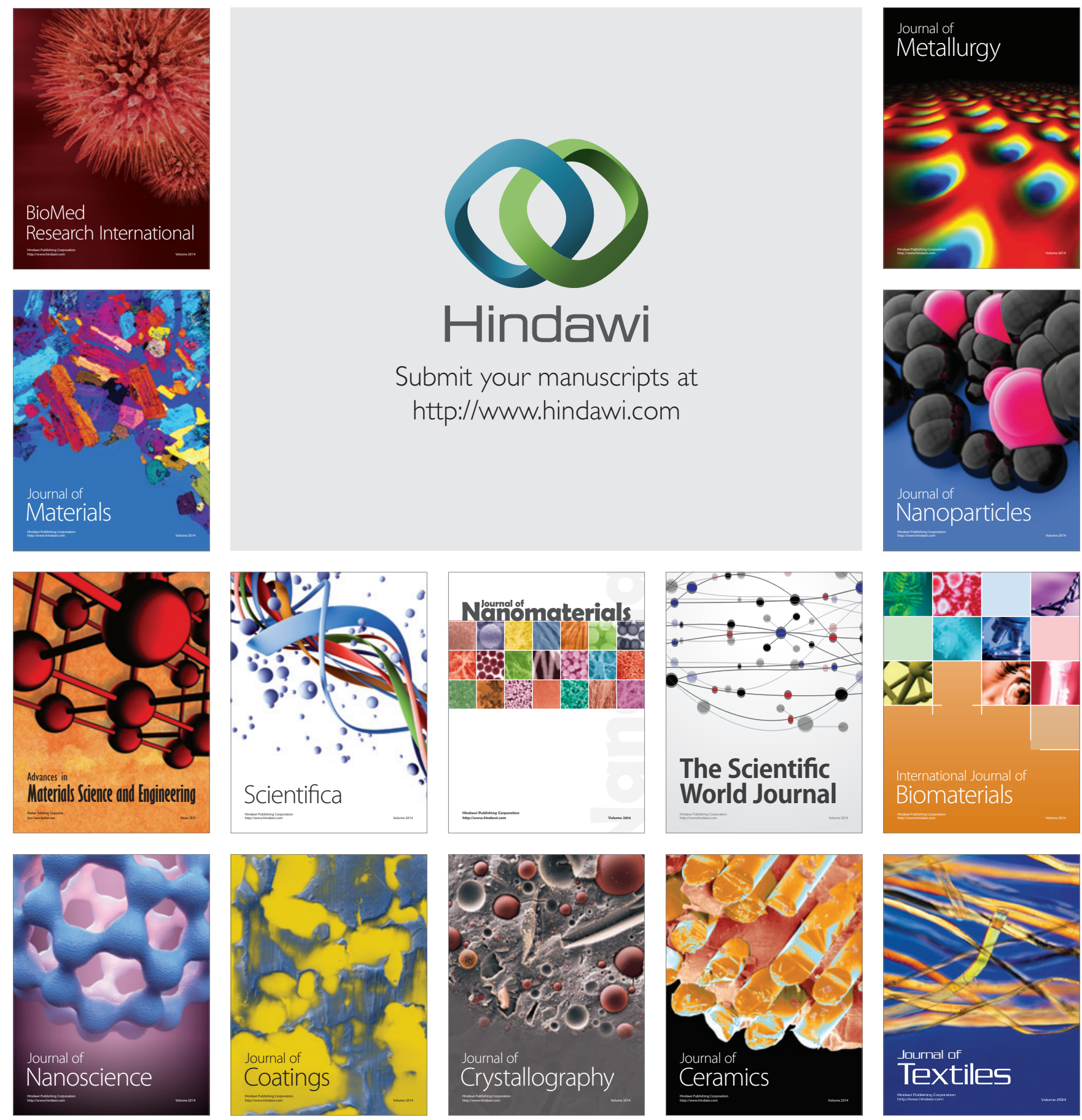